\title{
DATA ASSIMILATION FOR CONTROL OF A PLANE MIXING LAYER
}

\author{
N. Kumar*, F. Kerhervé, L. Cordier \\ Institut Pprime, CNRS - Université de Poitiers - ISAE-ENSMA, UPR 3346, \\ 11 Boulevard Marie et Pierre Curie, BP 30179, F86962 Futuroscope Chasseneuil Cedex, France \\ *Corresponding author: nishant.kumar@cnrs.pprime.fr
}

\begin{abstract}
The design of active model-based flow controllers requires the knowledge of a dynamical model of the flow. However, real-time and robust estimation of the flow state remains a challenging task when only limited spatial and temporal discrete measurements are available. In this study, the objective is to draw upon the methodologies implemented in meteorology to develop dynamic observers for flow control applications. Well established data assimilation (DA) method using Kalman filter is considered. These approaches are extended to both estimate model states and parameters. Simple non-linear dynamical models are first considered to establish quantitative comparisons between the different algorithms. An experimental demonstration for the particular case of a plane mixing layer is then proposed.
\end{abstract}

Keywords: data assimilation, active flow control, plane mixing layer

\section{Introduction}

Despite decades of intensive research in shape optimization, aerodynamic mechanisms such as separation and mixing still represent an important source of energy expenditure in transport vehicles. The manufacturers have consequently developed, over the years, a range of strategies to improve the aerodynamic performance of their vehicles. One of these strategies consists in inserting passive control devices like vortex generators directly in the flow-path. In general, these actuators are optimized considering the most likelihood inflow condition which potentially leads to bad performance for varying initial conditions and/or unsteady upstream perturbations. The classical remedy is then to consider active devices that can be actuated on and off based on the change of the inflow conditions. To drive such actuators, command laws and more globally valid controllers are required. To design these controllers, different control strategies can be employed. The class of model-based controllers is considered here. This approach requires the knowledge of a dynamical model of the flow that one wants to control in real-time. Experimentally, the identification of such a model is challenging because it is generally obtained from limited spatial and time discrete measurements.

This problem of state estimation is well-known in the field of meteorology where data is collected at several locations, with spatial and temporal scales varying in orders of magnitude, to estimate the evolution of the weather [7].
Classical methodologies involve the use of data assimilation (DA) techniques where data is assimilated, when available, with the dynamical model of weather forecast to correct the state estimate obtained by the model, and eventually, to correct the model itself for further, more accurate predictions. In the specific context of aerodynamicrelated problems, the complexity resides essentially in the large number of degrees of freedom of the flow dynamics, in the broad range of scales to examine and in nonlinearities driving the flow dynamics. To design a physical model that can be managed in real-time applications, the Navier-Stokes equations are traditionally projected on an appropriate reduced basis leading to a reduced-order model of the flow [6]. Proper Orthogonal Decomposition (POD) [2] is typically used to design the orthonormal basis but other decomposition methods can be employed to capture flow features which are believed essential to the desired objective. The derived physical reduced-order model (ROM) can then be used in conjunction with DA techniques to design dynamic observers able to predict the flow state from limited information [4].

In this work, such dynamic observers will be developed, building on the state-of-the-art in meteorology. The first part of the work covers the implementation of DA methods to numerical simulations. The second part will then focus on the experimental implementation by considering the canonical case of a plane mixing layer in the perspective of real-time control.

\section{Assessment of Data Assimilation method}

In this section, we evaluate a DA technique with the consideration of applicability to the experiments. Specifically, we will focus on a method known as Dual Ensemble Kalman Filter (Dual-EnKF) which belongs to a category of sequential data assimilation methods. Before discussing the method, we briefly discuss the dynamic model used in data assimilation for the optimal estimation of the state of the system. In agreement with the POD-ROM obtained by Galerkin projection of the Navier-Stokes equations onto the POD basis, the model equation corresponds to a quadratic polynomial [1] form given by

$$
\frac{d a_{i}(t)}{d t}=C_{i}+\sum_{j=1}^{N} L_{i j} a_{j}(t)+\sum_{j=1}^{N} \sum_{k=1}^{N} Q_{i j k} a_{j}(t) a_{k}(t)
$$

where $\mathbf{a}=\left(a_{1}, \cdots, a_{N}\right)^{T}$ is the state vector and may represent the POD temporal modes. The coefficients $C_{i}$, $L_{i j}$ and $Q_{i j k}$ are model's parameters which need to be tuned properly to describe the flow dynamics. 
Under forcing or varying inflow conditions, the model's parameters may change significantly. Both state and parameters estimation is therefore performed by the DualEnKF method. Dual-EnKF is a data assimilation method based on Kalman filter [3] where the state and parameters statistics are propagated in time (forecast step) to naturally provide the prior statistics for the assimilation of the next observations (analysis step). Due to finite ensemble size, the filter is subject to sampling errors, which must be adjusted to ensure stability of the scheme.

The data assimilation methods are evaluated based on their ability to: i) improve the description of the dynamics within the observation time window, and ii) provide a dynamical model which can correctly predict the longterm dynamics.

Dual-EnKF applied to $2 D$-cylinder wake flow The DA methods are applied to estimate the numerical $2 D$ cylinder wake flow at $R e=100$ obtained in the form of snapshots from a finite-element solver FreeFem++. The snapshots contain the information of the two components of the velocity vector $\mathbf{u}=[u, v]^{T}$. To build the reduced order model in (1), POD is first used to energetically extract the important features from the flow field. The first four modes which represent $99 \%$ of the energy are retained to build the ROM. However, we observe that for various reasons (structural instability of the Galerkin projection, truncation of the POD basis, inaccurate treatment of the boundary and pressure terms), this dynamical system does not represent sufficiently well the correct dynamics. This problem is tackled by Dual-EnKF method to obtain better approximation of the coefficients in the ROM and hence improve the ability to forecast the evolution of modes (see Fig. 1).

\section{Experimental Demonstration}

At the conference, we will also present results where data assimilation will be applied to experimental data obtained for a plane mixing-layer. The tests will be conducted in the closed-loop mixing layer wind-tunnel COCOM at Institut Pprime (Poitiers, France). The wind-tunnel features a twin-turbine design with two separate air streams, running in parallel and meeting in the test section to produce a mixing layer (see Fig. 2). The test section is $3 \mathrm{~m}$ long with a $1 \mathrm{~m} \times 1 \mathrm{~m}$ square cross-section. It begins at the throat of the convergent part of the wind tunnel, where the surface separating the two air streams terminates with a splitter plate. The splitter plate maintains a horizontal upper surface (from where the upper stream originates), while its lower surface is angled at 8 degrees so as to connect with the upper surface and form a relatively thin trail- ing edge. The splitter plate spans the entire width of the test section inlet, and extends by $39 \mathrm{~cm}$ into the test $\mathrm{sec}$ tion. The initial thickness of the splitter plate is $8 \mathrm{~cm}$ and the trailing edge is only $2 \mathrm{~mm}$ thick (allowing instrumentation of the plate for control purpose). The boundary layers at the end of the splitter plate are turbulent for operation at higher speeds.

Planar PIV will be performed and synchronised with a rake of 18 hot-wires located just downstream the PIV measurement plane as illustrated in Fig. 2. The PIV data will be used to identify a POD-based reduced-order model of the flow following the work of Cordier et al. [1]. The Dual-EnKF technique described in the previous section will be applied with the hot-wires data as the observation array in order to recover a time-resolved estimate of the flow field as well as an update of the model's parameters. A high-speed planar PIV data set will be used to validate the estimate.

\section{References}

[1] L. Cordier, B. Abou El Majd, and J. Favier. Calibration of POD Reduced-Order Models using Tikhonov Regularization. International Journal for Numerical Methods in Fluids, 2:63, 2010.

[2] J. L. Lumley. Atmospheric Turbulence and Wave Propagation. The structure of inhomogeneous turbulence, pages 166-178. Nauka, Moscow, 1967.

[3] H. Moradkhani, S. Sorooshian, H. V. Gupta, and P. R. Houser. Dual state-parameter estimation of hydrological models using ensemble Kalman filter. Advances in Water Resources, 28(2):135 - 147, 2005. ISSN 0309-1708.

[4] N. Papadakis. Assimilation de données images : application au suivi de courbes et de champs de vecteurs. Phd thesis, Université de Rennes I, 2007.

[5] L. Perret, E. Collin, and J. Delville. Polynomial identification of POD based low-order dynamical system. Journal of Turbulence, (7):N17, 2006.

[6] C. W. Rowley and S. T. M. Dawson. Model Reduction for Flow Analysis and Control. Annu. Rev. Fluid Mech., 49(1):387-417, 2017.

[7] O. Talagrand and P. Courtier. Variational Assimilation of Meteorological Observations With the Adjoint Vorticity Equation. I: Theory. Quarterly Journal of the Royal Meteorological Society, 113(478):13111328, 1987. 


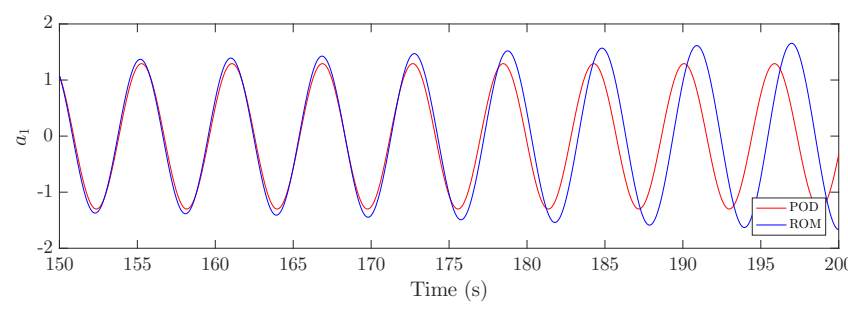

(a)

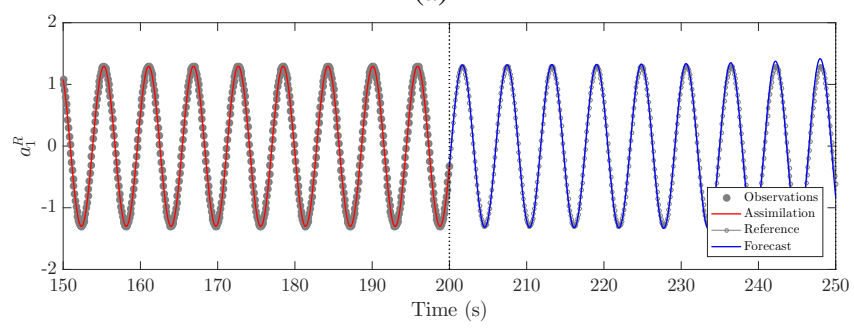

(c)

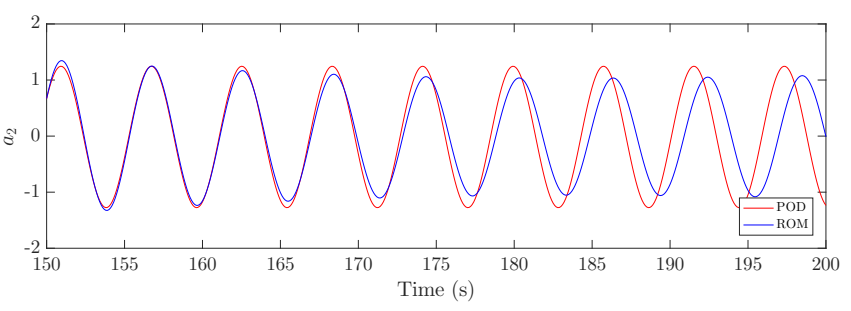

(b)

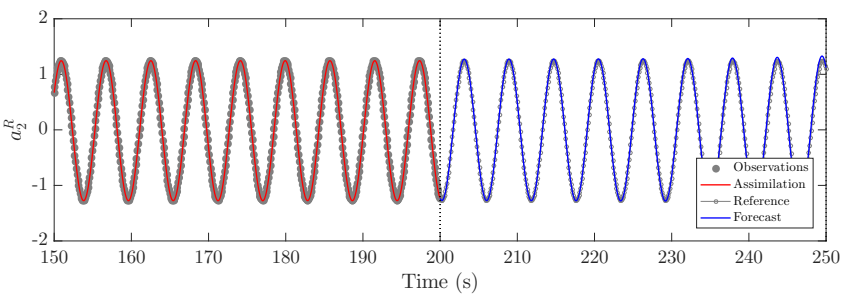

(d)

Figure 1: Time evolution of the POD-ROM for the temporal POD coefficients $a_{1}^{\mathrm{R}}$ and $a_{2}^{\mathrm{R}}$ corresponding to the two most energetic modes for the $2 D$-cylinder wake flow at $R e=100$. (a)-(b) ROM coefficients obtained from least mean-square estimation [5]. (c)-(d) ROM coefficients evaluated by Dual-EnKF. The dotted line at $t=200 \mathrm{~s}$ indicates the end of the assimilation window and beginning of the forecast window.

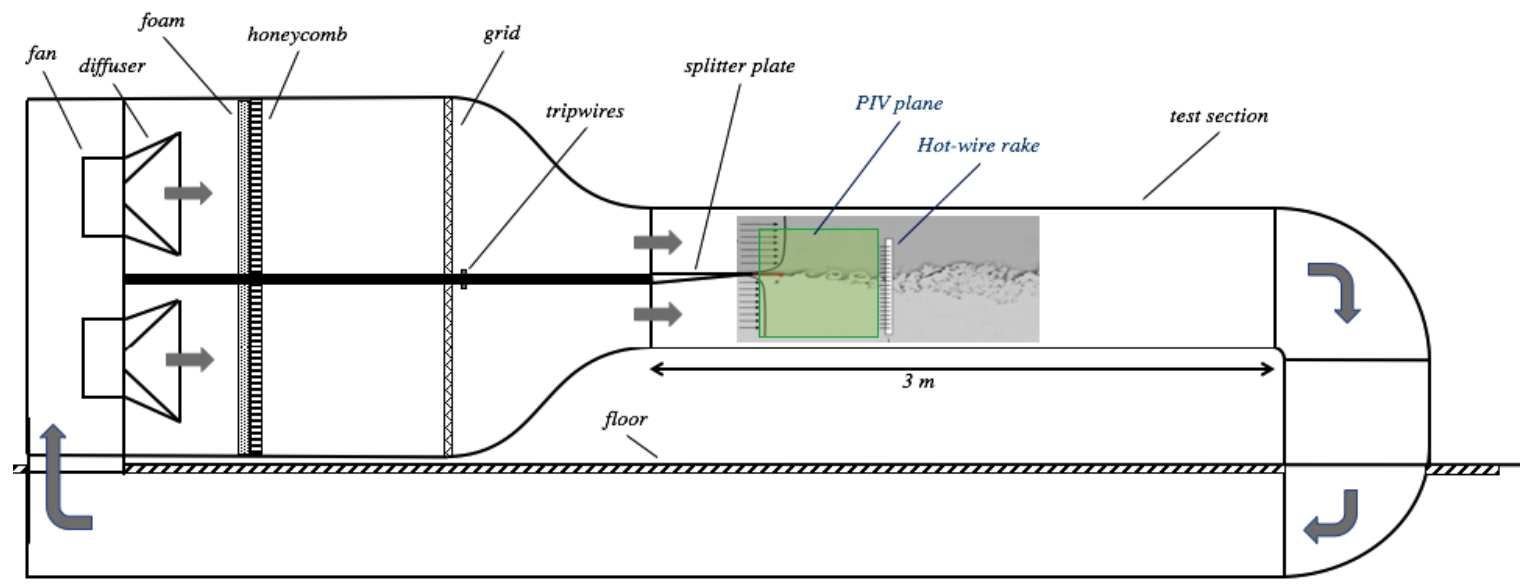

Figure 2: Schematic of the plane mixing layer experimental set-up. 